\title{
A Close Examination of Performance and Power Characteristics of 4G LTE Networks
}

\author{
Junxian Huang \\ University of Michigan \\ Z. Morley Mao \\ University of Michigan
}

\author{
Feng Qian \\ University of Michigan \\ Subhabrata Sen \\ AT\&T Labs - Research
}

\author{
Alexandre Gerber \\ AT\&T Labs - Research \\ Oliver Spatscheck \\ AT\&T Labs - Research
}

\begin{abstract}
With the recent advent of $4 \mathrm{G}$ LTE networks, there has been increasing interest to better understand the performance and power characteristics, compared with $3 \mathrm{G} / \mathrm{WiFi}$ networks. In this paper, we take one of the first steps in this direction.

Using a publicly deployed tool we designed for Android called 4GTest attracting more than 3000 users within 2 months and extensive local experiments, we study the network performance of LTE networks and compare with other types of mobile networks. We observe LTE generally has significantly higher downlink and uplink throughput than $3 \mathrm{G}$ and even $\mathrm{WiFi}$, with a median value of $13 \mathrm{Mbps}$ and $6 \mathrm{Mbps}$, respectively. We develop the first empirically derived comprehensive power model of a commercial LTE network with less than $6 \%$ error rate and state transitions matching the specifications. Using a comprehensive data set consisting of 5-month traces of 20 smartphone users, we carefully investigate the energy usage in $3 \mathrm{G}$, LTE, and WiFi networks and evaluate the impact of configuring LTE-related parameters. Despite several new power saving improvements, we find that LTE is as much as 23 times less power efficient compared with $\mathrm{WiFi}$, and even less power efficient than $3 \mathrm{G}$, based on the user traces and the long high power tail is found to be a key contributor. In addition, we perform case studies of several popular applications on Android in LTE and identify that the performance bottleneck for web-based applications lies less in the network, compared to our previous study in 3G [24]. Instead, the device's processing power, despite the significant improvement compared to our analysis two years ago, becomes more of a bottleneck.
\end{abstract}

\section{Categories and Subject Descriptors}

C.2.1 [Network Architecture and Design]: wireless communication; C.4 [Performance of Systems]: measurement techniques, modeling techniques, performance attributes; D.2.8 [Metrics]: Performance measures

\section{Keywords}

LTE, 4G, 4GTest, 3G, energy saving, power model simulation, network model simulation

Permission to make digital or hard copies of all or part of this work for personal or classroom use is granted without fee provided that copies are not made or distributed for profit or commercial advantage and that copies bear this notice and the full citation on the first page. To copy otherwise, to republish, to post on servers or to redistribute to lists, requires prior specific permission and/or a fee.

MobiSys'12, June 25-29, 2012, Low Wood Bay, Lake District, UK.

Copyright 2012 ACM 978-1-4503-1301-8/12/06 ...\$10.00.

\section{INTRODUCTION}

Initiated in 2004 by 3rd Generation Partnership Project (3GPP), the Long Term Evolution (LTE), commonly referred to as a type of $4 \mathrm{G}$ wireless service, aims at enhancing the Universal Terrestrial Radio Access Network (UTRAN) and optimizing radio access architecture [1]. Since 2009, LTE starts entering the commercial markets and is available now in more than 10 countries, with an expectedly fast-growing user base. The targeted user throughput is $100 \mathrm{Mbps}$ for downlink and 50Mbps for uplink, significantly higher than the existing $3 \mathrm{G}$ networks, with less than $5 \mathrm{~ms}$ user-plane latency [12]. Understanding the actual user-perceived network performance for LTE network and how it compares with its predecessor $3 \mathrm{G}$ and its competitors, e.g., WiFi and WiMAX, is important, yet not straightforward. Our previous study [24] takes one of the first steps to measure $3 \mathrm{G}$ network performance directly from end users. In this study, we follow up with a new tool, 4GTest, with enhanced measurement design and global server support, allowing us to characterize network performance of LTE and other mobile networks.

Besides higher bit rate, lower latency and many other service offerings for LTE, user equipment (UE) power saving is an important issue. LTE employs Orthogonal Frequency Division Multiplex (OFDM [15]) technology, which suffers from poor power efficiency. To save power, LTE uplink uses an special implementation of OFDM called SC-FDMA for uplink, with improved power efficiency. Discontinuous reception (DRX) has been employed by existing wireless mobile networks to reduce UE energy consumption. In UMTS [14], during the idle state, UE periodically wakes up to check paging messages and sleeps for the remaining time. LTE supports DRX for both RRC_CONNECTED and RRC_IDLE modes [16], seeking more opportunities to conserve UE battery. DRX is configured at a per-UE basis and controlled by a list of parameters in Table 2 The configuration of these parameters incurs tradeoff among UE power saving, channel scheduling delay, and signaling overhead.

To understand this tradeoff, existing studies use either total on time to estimate UE power usage [36 19], or a simplified LTE power model [25 34], which ignores the impact of downlink/uplink data rates. In this paper, we develop the first empirically derived comprehensive power model of a commercial LTE network, which accurately models UE energy usage. Also, existing studies 36 19, 25, 34] heavily rely on synthetic packet models instead of real user traces. Our study is the first that leverages a comprehensive real user data set, we call UMICH, consisting of 5-month traces of 20 smartphone users, to analyze the impact of LTE parameter configuration on realistic application usage patterns. UMICH data set only includes users traces in $3 \mathrm{G}$ and WiFi networks, but not in LTE network. We make our best efforts to overcome this limitation 


\begin{tabular}{|c|c|c|c|}
\hline$\S$ & Figures \& Tables & Data source & Description \\
\hline 4.1 & Figure $5 \mid 6$ & Public deployment of 4GTest & "Compare LTE network performance with other mobile networks \\
\hline 4.2 & Figure 7 & Local LTE Phone & Infer LTE parameters with network-based approach \\
\hline 4.3 .4 .4 & Figure 8 & LTE Laptop and LTE Phone & Understand the impact of various factors on LTE performance \\
\hline 5.15 .2 & 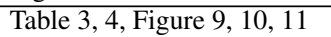 & Local LTE Phone & Derive LTE power model and compare with WiFi and $3 \mathrm{G}$ \\
\hline 5.3 & Figure 12 & Local LTE Phone & Compare bulk data transfer energy efficiency of LTE with WiFi and 3G \\
\hline 5.4 & Table 5 & Local LTE Phone & Validate LTE power model with real applications \\
\hline $6 . \overline{1} 6.2$ & Figure 14 & Simulation on UMICH data set & Analyze energy efficiency and the impact of different energy components \\
\hline 6.3 & 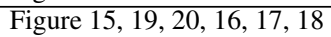 & Simulation on UMICH data set & Study the impact of LTE parameter configurations \\
\hline 7.1 & Figure 22 & Different local platforms & Compare JavaScript execution speed in 2009 and in 2011 \\
\hline 7.2 & Table 6 Figure 21 & Local LTE Phone & Study performance bottleneck of web-based applications \\
\hline
\end{tabular}

Table 1: Summary and index of experimental results.

by feeding WiFi traces into our LTE model simulation framework, given that RTT of WiFi is close to LTE (\$4.1) and throughput is less important than RTT for most web-based smartphone applications $(\$ 7.2)$.

Compared with 3G, LTE significantly improves the network performance. Meanwhile, device processing capability and software design have also improved remarkably over the last two years, comparing with our previous study [24]. To understand the potential performance bottleneck shift for smartphone applications, we perform case studies of several popular applications on Android. With the help of CPU, network and power traces, we devise a systematic methodology for application performance and power measurement and analysis.

We summarize the results of this paper in Table 1 and highlight our main contributions as follows.

(i) This paper is one of the first studies on commercial LTE networks. We characterize LTE network performance with both local experiments and global measurement by deploying a network performance measurement tool 4GTest with distributed server support globally. Generally, LTE is observed to have significantly higher downlink and uplink throughput than $3 \mathrm{G}$ and even $\mathrm{WiFi}$, with a median value of $13 \mathrm{Mbps}$ and $6 \mathrm{Mbps}$, respectively, which is even higher than the advertised throughput range of the LTE provider studied.

(ii) We develop the first empirically derived comprehensive power model of a commercial LTE network, considering both uplink and downlink data rates in addition to state transitions and DRX. It accurately quantifies the radio energy usage of UE with less than $6 \%$ error rate. Our power measurement also reveals that LTE is less power efficient than $3 \mathrm{G}$ and $\mathrm{WiFi}$ for small data transfers, e.g., for transferring one packet, the energy usage for LTE, $3 \mathrm{G}$ and WiFi is $12.76 \mathrm{~J}, 7.38 \mathrm{~J}$ and $0.04 \mathrm{~J}$, respectively. For bulk data transfer, LTE is more power efficient than $3 \mathrm{G}$, yet still lags behind WiFi, e.g., for downloading 10MB data, $3 \mathrm{G}$ and LTE require 34.77 and 1.62 times the energy of $\mathrm{WiFi}$, respectively. Considering that LTE is more energy efficient if fully utilized, it is even more crucial to optimize applications using tools like ARO [28] in LTE than 3G to achieve the full LTE savings. The energy model derived in this paper will provide the necessary understanding to drive those optimizations.

(iii) With the aforementioned power model, we build a tracedriven LTE analysis modeling framework, which breaks down the total energy consumption into different components, to identify the key contributor for energy usage. It also quantifies the channel scheduling delay perceived by UE and signaling overhead of cell towers. This analysis framework is applied to a comprehensive data set UMICH consisting 5 months' data from 20 real users. By comparing the estimated energy usage of different networks, LTE is as much as 23 times less power efficient compared with $\mathrm{WiFi}$, and even less power efficient than $3 \mathrm{G}$, despite the presence of DRX de- signed for energy saving. We also study the impact of configuring various LTE parameters on radio energy, channel scheduling delay, and signaling overhead. Similar to 3G UMTS network, LTE tail timer $T_{\text {tail }}$ remains a key parameter in determining UE energy usage and performance, as well as signaling overhead of cell towers.

(iv) We perform case studies of several popular applications on Android to understand the impact of improved LTE network performance and enhanced UE processing power on applications. The processing speed for contemporary smartphones have increased significantly compared with our analysis two years ago. However, we still identify that the performance bottleneck for web-based applications lies more in the device's processing power than in the network, indicated by the high average CPU usage of $79.3 \%$ in LTE network, as compared to $57.7 \%$ in $3 \mathrm{G}$ network, as well as the underutilized network capacity due to small object size in typical web transactions.

The remaining paper is organized as follows. We cover background on RRC and DRX for LTE in $\$ 2$ followed by experimental setup and methodology in $\$ 3$ We then present network characterization results, including 4GTest results in $\$ 4$ and power measurements in $\$ 5$ s summarizes the simulation analysis on UMICH data set, and \$7 includes the case study of several popular Android applications. We discuss related work in $\$ 8$ before concluding in $\$ 9$

\section{BACKGROUND}

We first cover the necessary background on LTE state machine behavior and corresponding power characteristics.

\subsection{Radio Resource Control (RRC) and Dis- continuous Reception (DRX) in LTE}

LTE has two RRC states, RRC_CONNECTED and RRC_IDLE [10 17], as shown in Figure 1. At RRC_CONNECTED state, UE can be in one of the three modes: Continuous Reception, Short DRX, and Long DRX. While at RRC_IDLE state, UE is only in DRX mode. Table 2 enumerates a list of important LTE parameters, which have significant impact on UE's radio energy consumption, user experience, and signaling overhead for cell towers. The terms in Table 2 are used consistently throughout this paper.

If UE is initially in RRC_IDLE state and receives/sends one packet, regardless of the packet size, a state promotion from RRC_IDLE to RRC_CONNECTED occurs with a relatively stable delay, similar to the promotion from IDLE to DCH/FACH in UTMS network [27]. We define the LTE promotion delay to be $T_{p r} 1$ consistently throughout this paper. During this period, radio resources are allocated to the UE.

After being promoted to RRC_CONNECTED, UE enters Continuous Reception by default and keeps monitoring the Physical Down-

\footnotetext{
${ }^{1} T_{\text {pro }}$ is a measured system property, different from the configurable LTE parameters in Table 2
} 


\begin{tabular}{|c|l|c|l|}
\hline Symbol & Full name & Measured value & Description \\
\hline \hline$T_{i}$ & DRX inactivity timer & $100 \mathrm{~ms}$ & UE stays in Continuous Reception for $T_{i}$ before DRX starts when idling \\
\hline$T_{i s}$ & Short DRX cycle timer & $20 \mathrm{~ms}$ & UE remains in Short DRX for $T_{i s}$ before entering Long DRX when idling \\
\hline$T_{t a i l}$ & RRC inactivity timer & $11.576 \mathrm{~s}$ & UE stays in RRC_CONNECTED for $T_{\text {tail }}$ before demoting to RRC_IDLE \\
\hline$T_{o n}$ & RRC_CONNECTED On Duration timer & $1 \mathrm{~ms}$ & The on duration of UE during each DRX cycle in RRC_CONNECTED \\
\hline$T_{\text {oni }}$ & RRC_IDLE On Duration timer & $43 \mathrm{~ms}$ & The on duration of UE during each DRX cycle in RRC_IDLE \\
\hline$T_{p s}$ & Short DRX cycle & $20 \mathrm{~ms}$ & The cycle period of Short DRX in RRC_CONNECTED \\
\hline$T_{p l}$ & Long DRX cycle & $40 \mathrm{~ms}$ & The cycle period of Long DRX in RRC_CONNECTED \\
\hline$T_{p i}$ & RRC_IDLE DRX cycle & $1.28 \mathrm{~s}$ & The cycle period of DRX in RRC_IDLE \\
\hline
\end{tabular}

Table 2: Important LTE RRC and DRX parameters.

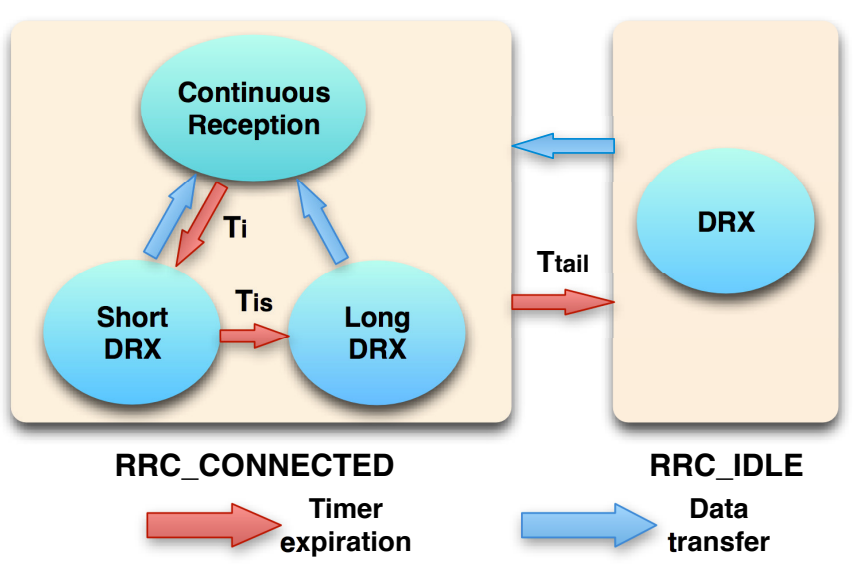

Figure 1: RRC state transitions in LTE network.

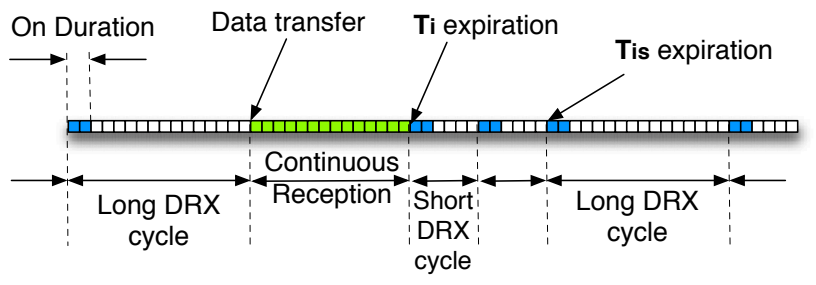

Figure 2: Illustration of the LTE DRX in RRC_CONNECTED.

link Control Channel (PDCCH), which delivers control messages to UE. UE also starts the DRX inactivity timer $T_{i}$, which is reset every time UE receives/sends a packet. Upon $T_{i}$ 's expiration without seeing any data activity, UE enters the Short DRX mode.

Discontinuous Reception (DRX) [16 30], illustrated in Figure 2 is adopted by LTE for UE to "micro-sleep" to reduce power consumption while providing high QoS and connectivity. DRX in RRC_CONNECTED and RRC_IDLE have similar mechanisms, but different parameter settings. A DRX cycle includes an On Duration during which the UE monitors PDCCH. UE rests for the rest of the cycle to save energy. The tradeoff between battery saving and latency is the guideline for determining the parameterization of DRX cycle. With a fixed On Duration, a longer DRX cycle reduces energy consumption of UE while increasing user-perceived delay, and a shorter DRX cycle reduces the data response delay at the cost of more energy consumption. Short DRX and Long DRX modes, having the same On Duration and differing in cycle length, are to meet these conflicting requirements.

When UE enters Short DRX, Short Cycle Timer $T_{i s}$ is started. Upon $T_{i s}$ 's expiration, if there is no data activity, UE switches to Long DRX; otherwise, UE goes back into Continuous Reception.

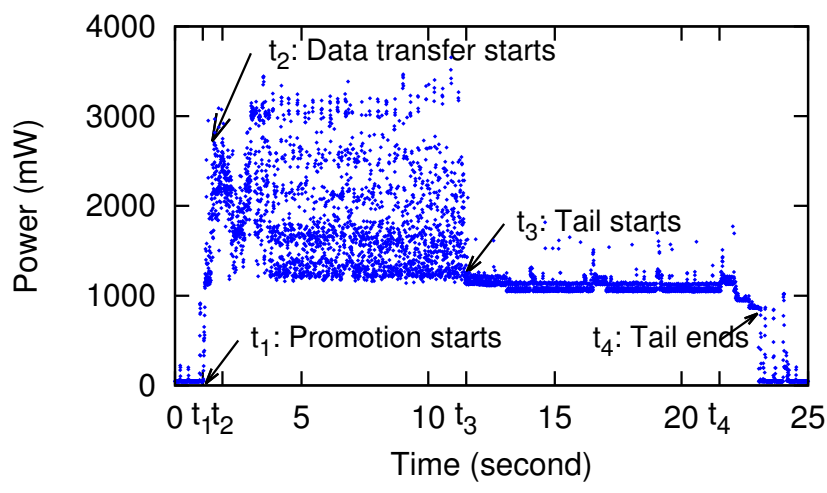

Figure 3: Power states of LTE.

For our measurement, $T_{i s}$ coincidentally equals $T_{p s}$, so only one cycle of Short DRX is expected to take place before $T_{i s}$ expires. Every time UE enters Continuous Reception when there is any data transfer, UE starts the tail timer $T_{\text {tail }}$, which is reset every time a packet is sent/received. When $T_{\text {tail }}$ expires, UE demotes from RRC_CONNECTED to RRC_IDLE and the allocated radio resource is released. Notice that $T_{\text {tail }}$ coexists with $T_{i}$ and $T_{i s}$.

\subsection{Smartphone power model for LTE}

Given the description of LTE state machine, we illustrate the power traces of an Android smartphone in a commercial LTE network based on local experiments described in $\$ 3.2$ We observe that network activities match the corresponding state transitions indicated by different power levels.

Figure 3 shows the power trace of uploading at the speed of $1 \mathrm{Mpb}$ for 10 seconds. With screen off, the energy is mostly consumed by the radio interfaces, as the power level is less than $20 \mathrm{~mW}$ before $t_{1}$. At $t_{1}$, the application sends a TCP SYN packet triggering RRC_IDLE to RRC_CONNECTED promotion, and the application waits for $T_{\text {pro }}$ until starting data transfer at $t_{2}$. Between $t_{2}$ and $t_{3}$, depending on the instant data rate, the power level fluctuates. We notice the power level during fast data transfer is significantly higher than the base power in RRC_CONNECTED, which motivates us to incorporate data rates into our LTE power model. After the data transfer completes at $t_{3}$, the device remains in RRC_CONNECTED for a fixed tail time $T_{\text {tail }}$, until $t_{4}$, when the device goes back to RRC_IDLE. The periodicity of DRX between $t_{3}$ and $t_{4}$ is not obvious due to limited sample rate.

In summary, this section provides necessary background information and some initial motivating observations for the following discussions on the network and power characterization of LTE network. 


\section{METHODOLOGY}

In this section, we present the methodology for network and power measurement, as well as trace-driven simulation analysis and real application case studies.

\subsection{Network measurement}

In this section, we discuss the design of our publicly deployed tool 4GTest, followed by experimental setup and methodology for local network measurement.

\subsubsection{The design of 4GTest}

Based on our previous experiences of developing 3GTest 6. 24], we design a new mobile network measurement tool for Android devices, called 4GTest [2], with higher accuracy and improved user experience. As a new feature, 4GTest allows users to switch among different network types, i.e., 3G, WiFi and LTE. We also improve the network measurement methodology leveraging the M-Lab [5] support. 4GTest server suite is deployed to 46 M-Lab nodes across the world, covering North America, Europe, Asia, and Australia. Each node has 4-core $2.00 \mathrm{GHz}$ Intel Xeon CPU and our virtual slice has 4GB memory and 100Mbps Ethernet network access, which ensures that the network bottleneck is unlikely on the wired network path.

RTT and variation test LTE has significantly smaller latency compared with $3 \mathrm{G}[12]$, hence the network distance between users and the measurement servers for the wired Internet path becomes less negligible. Given that the GPS coordinates for M-Lab nodes are known, in 4GTest, a nearest M-Lab node is selected for a user based on the current GPS location if available, or the IP address otherwise, with the help of a IP address to GPS coordinates mapping [4]. Such a mapping is sufficient for our purpose of finding coarse-grained location estimates for server selection.

To measure RTT and variation, 4GTest repeatedly establishes a new TCP connection with the server and measures the delay between SYN and SYN-ACK packet. Both the median of these RTT measurements and the variation are reported to our central server. Throughput test Since single-threaded TCP measurement is more sensitive to packet loss and hence less accurate [32], we use multi-threaded TCP measurement in 4GTest to estimate the peak channel capacity, i.e., three nearest server nodes in M-Lab are selected for each user at runtime to start concurrent threads for throughput test. Despite the shared nature of M-Lab nodes with other test tools, it is unlikely that all three selected nodes are overloaded in terms of CPU and network.

A throughput test lasts for 20 seconds, to balance across bandwidth usage, user waiting time and measurement accuracy. The initial 5 seconds are ignored empirically due to TCP slow start. The remaining 15 seconds are separated into 151 -second bins. The average throughput for each bin is calculated and the median of all bins is the measured throughput. Compared with using average throughput, median more accurately estimates the steady peak throughput by reducing the impact of abnormal bins, e.g., a very high bin due to initial buffering or a low bin due to temporary signal problem. Uplink and downlink tests share the same methodology.

\subsubsection{Local network measurement}

Devices for accessing LTE networks in this study are named LTE Phone and LTE Laptop. LTE Phone is an HTC phone with LTE data plan from a cellular ISP. It has 768 MB RAM memory and $1 \mathrm{GHz}$ Qualcomm MSM8655 CPU, running Android 2.2.1. LTE Laptop is a laptop equipped with LTE USB Modem. It has 8GB memory and $2.53 \mathrm{GHz}$ Intel Core Duo CPU, running Mac OS $\mathrm{X}$ 10.7.2.
Packet and CPU trace collection In order to collect packet traces on LTE Phone, we cross-compile tcpdump [9]. With a small snapshot length, only to capture TCP/UDP and IP headers, the CPU overhead of the background tcpdump process is less than 5\%. To capture CPU usage history, we write a simple C program to read /proc/stat in Android system every $25 \mathrm{~ms}$ and the overhead is verified to be smaller than $5 \%$ on LTE Phone.

Network-based approach for LTE parameter inference is used to validate the power-based approach $(\$ 3.2$. We use the following experiment to infer LTE state machine and RRC_IDLE DRX parameters. LTE Phone maintains a long lived TCP connection with a test server. For each experiment, server first sends a packet $P_{1}$ to trigger UE's promotion to RRC_CONNECTED, and after $X$ seconds, server sends another packet $P_{2}$ to the phone to measure RTT. Assume the RTT at RRC_CONNECTED is $R T T_{b}$ ignoring the minor impact of DRX in RRC_CONNECTED. When $X \leq T_{\text {tail }}$, $R T T(X)=R T T_{b}$. Otherwise, $R T T(X)=R T T_{b}+T_{\text {pro }}+T_{d r x}$, where $T_{d r x}=T_{n}-X$ and $T_{n}$ is the start time of the next DRX on duration after $P_{2}$ 's arrival. If $P_{2}$ 's arrival is inside any DRX on duration, $T_{d r x}=0$.

To understand the impact of packet size on one-way delay (OWD), uplink and downlink OWD is measured with varying packet size. For each packet size, 100 samples are measured, and we make sure that packets are not fragmented. OWD is measured between LTE Laptop and a test server. A GPS Receiver [3] is connected to LTE Laptop, which uses the received GPS messages for time synchronization. The test server is also time-synced with another GPS clock. The maximum error of our setup is less than $1 \mathrm{~ms}$.

We compare LTE with $3 G$ and WiFi by local experiments on LTE Phone. When $4 \mathrm{G}$ is disabled in LTE Phone, it connects to $3 \mathrm{G}$ network and the network type is eHRPD. For WiFi, with the data network radio interface turned off, LTE Phone connects to a wireless router, Cisco-Linksys WRT54GL Wireless-G Broadband Router, 802.11g in channel 2.437GHz. The Internet ISP for WiFi is Comcast and the advertised downlink peak data rate is $6 \mathrm{Mbps}$.

\subsection{Power measurement}

Similar to previous studies [27 35], we use Monsoon power monitor [7] as power input for LTE Phone measuring power traces at the same time. The power trace contains two fields, timestamp and average instant power, and the sampling rate is $5000 \mathrm{~Hz}$. We share the same observation with previous study [35] that screen plays an important role in device power consumption, i.e., with screen $100 \%$ on, the UE idle power is $847.15 \mathrm{~mW}$ compared with $11.36 \mathrm{~mW}$ with screen off. For all measurements, we keep the test application running in the background with screen completely off to minimize power noise, unless UI interactions are required and screen should be kept on, i.e., measuring power for browser. In this case, we subtract screen power from the total, with slightly increased noise. All experiments are repeated at least 5 times to reduce measurement error.

To measure state transition power levels, UE keeps a long-lived TCP connection with the server and packet traces are collected to make sure there is no background traffic. In order to trigger state promotions, we make the device idle for sufficient time, e.g., 30 seconds, and then send a packet from server to client. UE remains idle afterwards and demotes to idle state in the end, and the power trace covers the full tail.

\subsection{Trace-driven analysis}

To compare energy consumption for different networks using real user traces and evaluate the impact of setting LTE parameters, we devise a systematic methodology for trace-driven analy- 
sis, which is applied to a comprehensive user trace data set, named UMICH.

\subsubsection{UMICH data set for analysis}

$\mathrm{UMICH}$ data set is collected from 20 smartphone users for five months, 05/12/2011 10/12/2011, totaling 118 GB. These participants consist of undergraduate and graduate students from 8 departments at University of Michigan. The 20 participants are given Motorola Atrix (11 of them) or Samsung Galaxy S smartphones (9 of them) with unlimited voice, text and data plans, all running Android 2.2. As stated in our study consent form, we keep collected data and users' identities strictly confidentia ${ }^{2}$

Custom data collection software developed by us are deployed on the 20 smartphones. It continuously runs in the background and collects full packet traces in tcpdump format including both headers and payload. Both cellular and Wi-Fi traces are collected without any sampling performed. The data collector incurs no more than $15 \%$ of CPU overhead, although the overhead is much lower when the throughput is low (e.g., less than $200 \mathrm{kbps}$ ).

We also build a data uploader that uploads the data to our server when the phone is idle as indicated by low network throughput. The upload is suspended by detected network activity of user applications. The entire data collection and uploading process is transparent to the users.

\subsubsection{Trace-driven modeling methodology}

We build a network model and power model analysis framework for the trace-driven analysis, totaling about 8,000 lines of code in $\mathrm{C}++$.

Network model simulator takes the binary packet trace files in libpcap [9] format and preprocesses the data following the same methodology as previous study [27], for the purpose of removing existing delays imposed by state promotions and extracting the actual traffic patterns, e.g., for the purposing of simulating the trace in another network. It then applies a specific network model to the processed traces and adjusts timestamps for some packets, since different network models have different delay behavior due to state machine differences. There are two reasons for a packet's timestamp to be adjusted, promotion delay and DRX waiting time. We use the same methodology to adjust timestamp for promotion delay as previous study [27]. In LTE network, if there is no packet for over $T_{\text {tail }}$ time, when the next packet $P$ arrives, an extra delay $T_{\text {pro }}$ is inserted before $P$. In terms of DRX waiting time, if a downlink packet $P^{\prime}$ comes at time $T_{P^{\prime}}$ and UE is in DRX mode, either in RRC_CONNECTED or RRC_IDLE, there is no DRX waiting time if $P^{\prime}$ arrives inside any DRX on duration; otherwise, $P^{\prime}$ would experience an extra delay of $T_{n}-T_{P^{\prime}}$, where $T_{n}$ is the start time of the next DRX on duration after $T_{P^{\prime}}$. The parameters for different network models are listed in Table 3 obtained from local experiments. We also empirically assume that UE goes into complete sleep with 0 power usage after being idle for $T_{\text {tail } 2}(1$ minute $)$. Notice that $T_{\text {tail2 } 2}$ is not actually observed in our experiments and is only used to bound the total idle energy and simplify our analysis.

The output of network model simulator is an array of packets with adjusted timestamps, in ascending order, as well as the RRC and DRX states of UE at any time.

Power model simulator takes the output of network model simulator and calculates the energy consumption based on the power model, detailed in $\$ 5$ We break down the total energy into four components: promotion, data transfer, tail, and idle.

\footnotetext{
${ }^{2}$ This user study has been approved by the University of Michigan IRB-HSBS \#HUM00044666.
}

For promotion energy, both promotion delay $T_{\text {pro }}$ and average promotion power $P_{\text {pro }}$ are fixed. If a trace includes $N$ promotions, promotion energy is given by $N T_{\text {pro }} P_{\text {pro }}$.

For idle energy in RRC_IDLE, assume that DRX cycle is $T_{p i}$ (with base power $P_{b}$ ) and on duration is $T_{\text {oni }}$ (with on power $P_{\text {oni }}$ ), energy consumption of an duration of $t_{i d l e}$ is given by

$$
\left\lfloor\frac{t_{\text {idle }}}{T_{p i}}\right\rfloor\left(T_{\text {oni }} P_{\text {oni }}+\left(T_{p i}-T_{\text {oni }}\right) P_{b}\right)+E_{\text {res }}
$$

where the remaining energy $E_{\text {res }}$ can be calculated as

$$
E_{\text {res }}= \begin{cases}T_{\text {res }} P_{\text {oni }} & \text { if } \quad T_{\text {res }} \leq T_{\text {oni }} \\ T_{\text {oni }} P_{\text {oni }}+\left(T_{\text {res }}-T_{\text {oni }}\right) P_{b} & \text { if } \quad T_{\text {res }}>T_{\text {oni }}\end{cases}
$$

with

$$
T_{\text {res }}=t_{\text {idle }}-T_{p i}\left\lfloor\frac{t_{i d l e}}{T_{p i}}\right\rfloor
$$

Tail energy is more complex for LTE since RRC_CONNECTED has three modes as in Figure 1 while $3 \mathrm{G}$ and WiFi do not have DRX in connected mode. We simulate the transitions among Continuous Reception, Short DRX and Long DRX to calculate tail energy, in a similar way of idle energy calculation. Note that we only count tail energy when the tail is complete, otherwise, the energy is considered as data transfer energy, i.e., if one packet $P$ is observed before UE demotes to RRC_IDLE at the end of the tail, the energy between $P$ and the previous packet is still considered as data transfer energy.

For data transfer energy, we propose a novel model. Assume uplink throughput is $t_{u}$ (Mbps) and downlink throughput is $t_{d}$ (Mbps), the instant power level $(\mathrm{mW})$ of UE is

$$
P=\alpha_{u} t_{u}+\alpha_{d} t_{d}+\beta
$$

Validation of this formula is in $\$ 5.2$ We simply consider payload throughput in our power model, without considering header length and ACK packets due their small impact. Since TCP instant throughput changes frequently, in order to accurately estimate the instant power levels, we divide the trace into small time windows with size $W$ seconds and within each window, throughput $t_{u}$ and $t_{d}$ are calculated, which determine the power $P . W$ is set to be 1 second empirically in our setup and the total energy is not sensitive to the choice of $W$ as long as $W$ is larger than a few RTTs and smaller than most flow durations.

\subsection{Application performance}

As a case study, we select a few smartphone applications and collect both network and CPU traces on LTE Phone. Specifically, we select the default browser, YouTube, NPR News and Android Market as our sampled applications given their popularity. For the default browser, we choose two different usage scenarios, i.e., visiting mobile version of google.com representing a simple website and visiting non-mobile version of yahoo. com representing a content-rich website. We name the two browser usage scenarios as Website G and Website Y, respectively. While for the other applications, we click the application icon until the initial page is fully presented to the user. We make sure the cache is cleared before each experiment.

Figure 4 shows co-located network and CPU trace of Website $\mathrm{Y}$. At time 0 , when GO button is clicked, loading starts. Before $t_{a}$, CPU usage stays low most of the time given that UE has not finished downloading the HTML or JavaScript objects. Starting from $t_{a}$, CPU usage jumps to $100 \%$ and remains a high average usage until $t_{c}$. We notice that network activity nearly stops after $t_{b}$, with only a few TCP FIN packets for closing TCP connections afterwards, some even come seconds later, e.g., at time 10.5 seconds. 


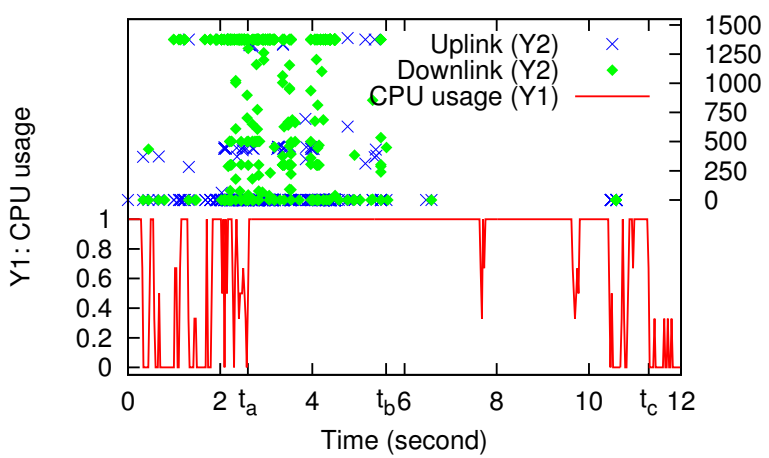

Figure 4: Network and CPU trace for Website $Y$ in LTE.

During the time between $t_{a}$ and $t_{c}$, UE is rendering HTML pages or executing JavaScript, and during the time between $t_{a}$ and $t_{b}$, UE is also downloading web objects in parallel.

We define $t_{c}$ to be the application loading time instead of $t_{b}$, since at $t_{b}$, UE has not fully rendered the contents for the user, though the download process is complete. We validate this by collecting video traces with a camcorder facing the screen of the phone. We replay the video at normal speed and manually mark the start time and the end time, when the website gets fully loaded and all UI activity indicators stop. We verify that $t_{c}$ is an accurate estimate for application loading time. We also define the average CPU usage between time 0 and $t_{c}$ to be the CPU usage for this application.

Compared with a previous approach for measuring web browsing performance by modifying and instrumenting WebKit [33], our approach is more lightweight and easily extensible to other applications other then web browsers, with reasonable accuracy. One limitation of our approach is that it is not applicable for applications, e.g., streaming video/audio and some game applications, whose CPU usage remains high after initial loading.

\section{LTE NETWORK CHARACTERIZATION}

We present network measurement results from publicly deployed 4GTest in this section. In addition, we carry out extensive local measurement on an LTE network to infer RRC and DRX parameters, and understand the impact of various factors on network performance.

\subsection{Comparing LTE to other mobile networks}

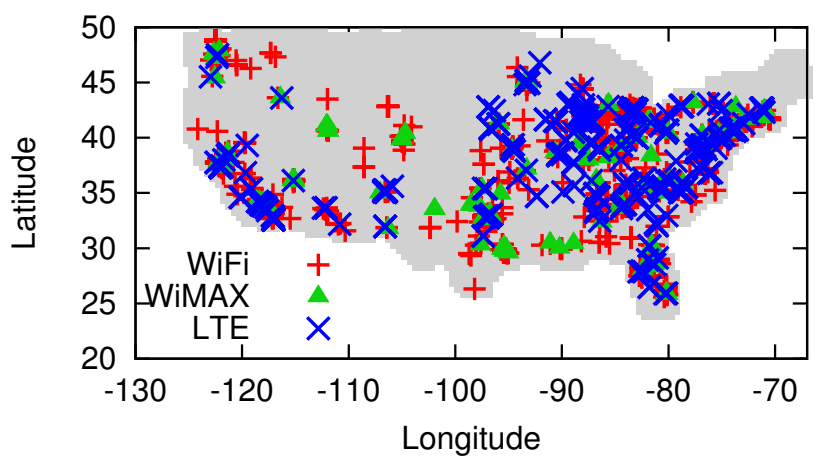

Figure 6: 4GTest user coverage in the U.S.

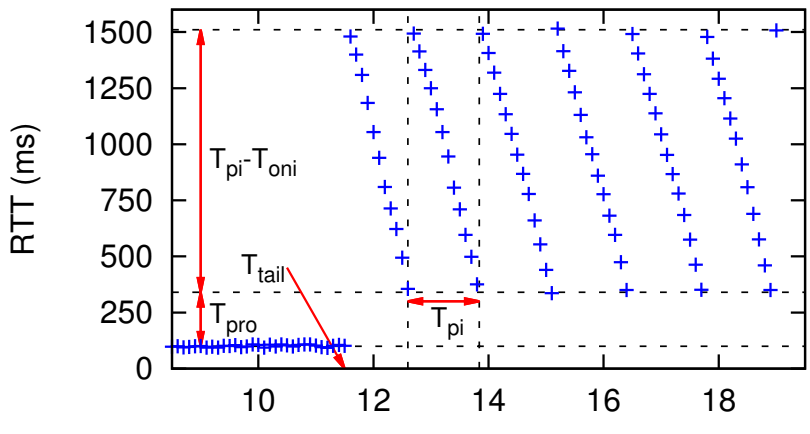

Idle time between packets (second)

Figure 7: LTE RTT v.s. inter-packet idle time.

4GTest was initially released on Android Market on 10/15/2011. Till 12/15/2011, there has been altogether 3294 global users and 13768 runs. In the analysis, we focus on 2292 users and 7063 runs inside the U.S., among which 380 are LTE users with 982 runs. We check the distribution of 4GTest users for different network types. For example, in Figure 6 the coverage of LTE, WiMAX, and WiFi are mostly similar, covering 39, 37 and 44 states in the U.S., respectively. We also observe that other networks have similar coverage across the U.S. This indicates that 4GTest data set enables fair comparison on the distribution of performance metrics for different mobile networks in the U.S.

Figure 5 summarizes the performance comparison among various mobile networks based on 4GTest data set. We present anonymized data based on the technology type. The box plot for each metric shows the 95th, 75th, 50th, 25th and 5th percentile among all measurements for each network type. We observe that LTE network has a high downlink and uplink throughput, with the median to be $12.74 \mathrm{Mbps}$ and 5.64Mbps, respectively, which is much higher than WiFi's $4.12 \mathrm{Mbps}$ (downlink) and $0.94 \mathrm{Mbps}$ (uplink), as well as 4.67Mbps (downlink) and 1.16Mbps (uplink) for WiMAX. The 3G family, including eHRPD, EVDO_A and HSDPA clearly lag behind LTE. We also observe relatively high variation on LTE throughput for different users at different locations, and even for the same user at the same location across different runs.

In terms of RTT and RTT jitter, LTE with the median RTT $69.5 \mathrm{~ms}$ and RTT jitter 5.6ms is comparable to WiFi's $64.5 \mathrm{~ms}$ (RTT) and 7.9ms (RTT jitter). Similar to throughput comparison, WiMAX also lags behind LTE with median RTT to be $125.0 \mathrm{~ms}$ and RTT jitter to be $21.0 \mathrm{~ms}$. For $3 \mathrm{G}$ networks, compared with our previous study [24], median RTT has been reduced from a median of $400 \mathrm{~ms}$ to below $200 \mathrm{~ms}$, some even has a median of 78ms (HSDPA 2), close to LTE RTT. RTT jitter for $3 \mathrm{G}$ networks are all larger than $10 \mathrm{~ms}$.

To summarize, Figure 5 provides a fair comparison on performance distribution among different mobile networks, from which we observe that LTE significantly improves network throughput as well as RTT and jitter, making it comparable to or even better than WiFi for throughput. WiMAX lags behind LTE, followed by HSDPA, eHRPD and EVDO_A networks.

\subsection{Network-based LTE parameter inference}

Figure 7 summarizes the network-based approach for LTE state machine and DRX parameter measurement using LTE Phone, with the methodology discussed in $\$ 3.1 .2$ Each point is the median of five runs and for each run, RTT is measured after making LTE Phone idle for a specific time. The pattern of RTT is expected as inter- 


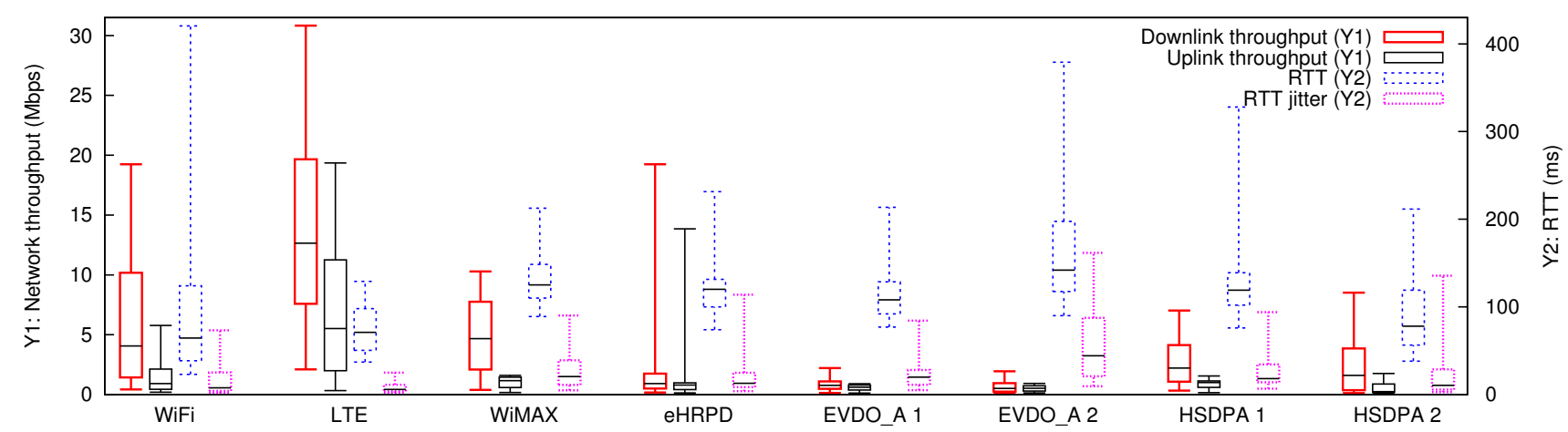

Figure 5: 4GTest result analysis based on network type ${ }^{\star}$.

${ }^{\star}$ Each network type corresponds to the data of one cellular ISP, except for WiFi which has data of various ISPs.

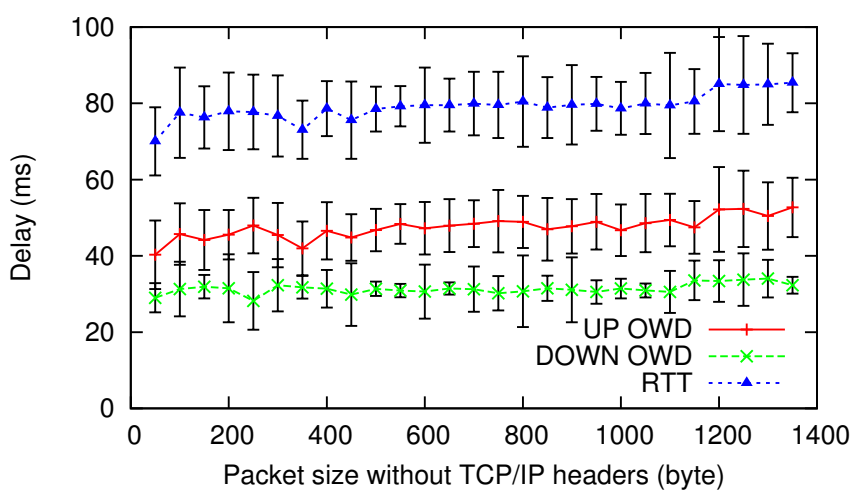

Figure 8: OWD/RTT v.s. packet size in LTE network.

packet idle time changes. Based on these results, we can infer the values of the following parameters in Table $2 T_{\text {tail }}$ is around $11.5 \mathrm{~s}$, $T_{p i}$ is $1.28 \mathrm{~s}$ and $T_{\text {oni }}$ is close to $45 \mathrm{~ms}$. In addition, we observe $T_{p r o}$ is around $260 \mathrm{~ms}$. These measured values agree with those measured from the power-based approach in Table 3 , cross-validating our measurement methodology.

\subsection{One-way delay and impact of packet size}

With the setup in $\$ 3.1 .2$ we measure uplink/downlink one-way delay (OWD) for both LTE and WiFi networks. Experiment in WiFi shows that both uplink and downlink OWD are around 30ms with little correlation with packet size, and RTT is stable around $60 \mathrm{~ms}$. However, for LTE, Figure 8 shows that uplink OWD is clearly larger than downlink OWD. We also observe that uplink OWD for LTE slightly increases as packet size grows, while the median of downlink OWD stays more stable around $30 \mathrm{~ms}$. The median RTT ranges from $70 \mathrm{~ms}$ to $86 \mathrm{~ms}$ as packet size increases. In summary, RTT in LTE is more sensitive to packet size than $\mathrm{WiFi}$, mainly due to uplink OWD.

\subsection{Mobility}

Requirements for LTE [12] specifies that mobility across the cellular network should be supported and optimized for low mobile speed from $0 \sim 15 \mathrm{~km} / \mathrm{h}$. Higher mobile speed of $15 \sim 120 \mathrm{~km} / \mathrm{h}$ should be supported with high performance. In this paper, we carry out one of the first studies to quantify the impact of mobile speed for a commercial LTE network.

We measure RTT and downlink/uplink throughput with 4GTest at three different mobile speeds: stationary, $35 \mathrm{mph}$ and $70 \mathrm{mph}$, highway speed limit. All experiments are done in the same car for fair comparison. Given that 4GTest takes less than 2 minutes to finish, handover seldom happens during a single test and we filter out samples with cell ID changed during the test. Previous work [29] has shown that signal strength varies across locations and has a non-negligible impact on performance, so we only select sample points with the similar signal strength for fair comparison.

We observe that RTT remains stable at different speeds, with small variation. Uplink and downlink throughput both have high variation of $3 \sim 8 \mathrm{Mbps}$. Comparing throughput at different speeds, there is no clear difference, given the high variation. Our experiments show that at least at our test location, there is no major performance downgrade at high speed for LTE, satisfying the requirements in [12].

In addition, we also study the correlation between LTE performance and time of day. We periodically run 4GTest for 48 hours in two randomly selected days. RTT's median value remain stable at $68 \mathrm{~ms}$ across different hours. Notice that we do not use the 4GTest results from real users to study this time of day pattern, because those results may also contain user-specific and location-wise factors which affect the performance and are hard to be excluded. For downlink and uplink throughput, variation across different hours is observed; however, there is no strong correlation with time of day. This may imply that, at our location, LTE currently does not have too many users and has well-provisioned resources available.

To summarize, with both global experiments, via 4GTest, and local experiments, we observe that LTE has significantly improved network performance over $3 \mathrm{G}$, making it comparable to, if not better than, WiFi.

\section{LTE POWER MODEL CONSTRUCTION}

This section summarizes the construction of the new LTE power model, as well as the $3 \mathrm{G}$ and WiFi model measured from the same LTE Phone. We then compare energy efficiency in bulk data transfer for different networks and validate LTE power model in the end.

\subsection{Power model for RRC and DRX}

With the experimental setup described in $\$ 3.2$ we measure power model for LTE, 3G, and WiFi on LTE Phone, summarized in Ta- 


\begin{tabular}{|c|c|c|c|}
\hline & $\begin{array}{c}\text { Power }^{\star} \\
(\mathrm{mW})\end{array}$ & $\begin{array}{c}\text { Duration } \\
(\mathrm{ms})\end{array}$ & $\begin{array}{c}\text { Periodicity } \\
(\mathrm{ms})\end{array}$ \\
\hline \hline Screen off (base) & $11.4 \pm 0.4$ & N/A & N/A \\
\hline Screen 100\% on & $847.2 \pm 2.7$ & N/A & N/A \\
\hline \hline LTE promotion & $1210.7 \pm 85.6$ & $\begin{array}{c}T_{p r o}: \\
260.1 \pm 15.8\end{array}$ & N/A \\
\hline $\begin{array}{c}\text { LTE Short DRX On } \\
\text { RRC_CONNECTED }\end{array}$ & $1680.2 \pm 15.7$ & $\begin{array}{c}T_{\text {on }}: \\
1.0 \pm 0.1\end{array}$ & $\begin{array}{c}T_{p s}: \\
20.0 \pm 0.1\end{array}$ \\
\hline $\begin{array}{c}\text { LTE Long DRX On } \\
\text { RRC_CONNECTED }\end{array}$ & $1680.1 \pm 14.3$ & $\begin{array}{c}T_{\text {on }}: \\
1.0 \pm 0.1\end{array}$ & $\begin{array}{c}T_{p l}: \\
40.1 \pm 0.1\end{array}$ \\
\hline LTE tail base & $1060.0 \pm 3.3$ & $\begin{array}{c}T_{\text {tail }}: \\
11576.0 \pm 26.1\end{array}$ & N/A \\
\hline $\begin{array}{c}\text { LTE DRX On } \\
\text { RRC_IDLE }\end{array}$ & $594.3 \pm 8.7$ & $\begin{array}{c}T_{\text {oni }}: \\
43.2 \pm 1.5\end{array}$ & $\begin{array}{c}T_{p i}: \\
1280.2 \pm 7.1\end{array}$ \\
\hline \hline 3G promotion & $659.4 \pm 40.4$ & $582.1 \pm 79.5$ & N/A \\
\hline 3G DCH tail base & $803.9 \pm 5.9$ & $8088.2 \pm 149.6$ & N/A \\
\hline 3G FACH tail base & $601.3 \pm 6.4$ & $824.2 \pm 148.1$ & N/A \\
\hline 3G DRX (idle) & $374.2 \pm 13.7$ & $55.4 \pm 1.5$ & $5112.4 \pm 37.7$ \\
\hline \hline WiFi promotion & $124.4 \pm 2.6$ & $79.1 \pm 15.1$ & N/A \\
\hline WiFi tail base & $119.3 \pm 2.5$ & $238.1 \pm 9.2$ & N/A \\
\hline WiFi beacon (idle) & $77.2 \pm 1.1$ & $7.6 \pm 0.1$ & $308.2 \pm 1.0$ \\
\hline
\end{tabular}

Table 3: LTE, 3G, and WiFi power model.

\footnotetext{
* All power readings in this table include the base power (screen off), which has negligible impact on total energy.
}

ble 3 The LTE parameter values are validated by the networkbased measurement in $\$ 4.2$ For simplicity, we ignore the WiFi AP scanning and association, assuming UE is already connected with an AP.

First, we observe that LTE reduces the promotion delay $\left(T_{\text {pro }}\right)$ from 3G's $582.06 \mathrm{~ms}$ to $260.13 \mathrm{~ms}$. However, the power level is almost doubled, i.e., $1210.74 \mathrm{~mW}$ (LTE) v.s. $659.43 \mathrm{~mW}$ (3G). WiFi has the most lightweight state promotion with smaller $T_{\text {pro }}$ and much lower power level.

Secondly, LTE appears to have longest tail (11.576 seconds) with highest tail base power $(1060.04 \mathrm{~mW})$. Summing up DCH and FACH tail, 3G's total tail time (8.9 seconds) is smaller than LTE's $T_{\text {tail }}$ of 11.6 seconds. Even 3G DCH's tail base power is $24.17 \%$ lower than LTE's tail base power, and the gap becomes $25.25 \%$ if we consider LTE DRX in RRC_CONNECTED with a high on duration power $(1680.20 \mathrm{~mW})$. WiFi is much more power efficient, with shorter tail and much lower base power.

We also compare LTE DRX in RRC_IDLE with 3G DRX and WiFi beacon in the idle state. LTE has the highest on power and slightly smaller On Duration than 3G, while WiFi has smallest on power and On Duration. The cycle of LTE (1.28 seconds) is in between $3 \mathrm{G}$ and WiFi.

Based on these observations, LTE is less energy efficient during idle state and for transferring smaller amount of data. For example, if only one packet is transferred, the energy usage considering both promotion and tail energy for LTE, $3 \mathrm{G}$ and WiFi is $12.76 \mathrm{~J}$, $7.38 \mathrm{~J}$ and $0.04 \mathrm{~J}$, respectively. One possible reason for LTE's higher power states is that devices must incorporate multiple-input and multiple-output (MIMO) to support LTE network, e.g., the test device we use has 1 transmit antenna and 2 receive antennas, which contributes to higher power consumption.

\subsection{Power model for data transfer}

Previous work on 3G UMTS power modeling either treats DCH power state to have a fixed power value [35 28], or assumes energy per bit to be the same constant for both uplink and down-

\begin{tabular}{|c|c|c|c|c|}
\hline & $\alpha_{u}(\mathrm{~mW} / \mathrm{Mbps})$ & $\alpha_{d}(\mathrm{~mW} / \mathrm{Mbps})$ & $\beta(\mathrm{mW})$ & $\alpha_{u} / \alpha_{d}$ \\
\hline LTE & 438.39 & 51.97 & 1288.04 & 8.44 \\
\hline 3G & 868.98 & 122.12 & 817.88 & 7.12 \\
\hline WiFi & 283.17 & 137.01 & 132.86 & 2.07 \\
\hline
\end{tabular}

Table 4: Data transfer power model.

link [18]. These assumptions might be reasonable given that $3 \mathrm{G}$ has relatively low data rates. However, for LTE, we observe that device power is much higher during high speed data transmission (up to $3300 \mathrm{~mW}$ for uplink) relative to the base power $(1060 \mathrm{~mW}$ ) in RRC_CONNECTED, and there is significant difference between downlink and uplink power levels at the same data rate. In this paper, we propose a new comprehensive power model for LTE empirically derived in a commercial LTE network.

We start with measuring device power states with controlled uplink or downlink throughput. The impact of TCP ACK packets, which are small in size, is minor, thus ignored.

Figures 9 and 10 present the power-throughput curve for LTE, $3 \mathrm{G}$, and WiFi. The curves are limited by the peak data rate we can achieve at the test location. We observe that for all networks, a linear model fits well for both uplink and downlink. Assume uplink throughput is $t_{u}$ (Mbps) and downlink throughput is $t_{d}$ (Mbps), the power level (mW) for uplink is $P_{u}=\alpha_{u} t_{u}+\beta$ and for downlink $P_{d}=\alpha_{d} t_{d}+\beta$. The best fit parameters are listed in Table 4

By looking at $\alpha_{u} / \alpha_{d}$, we notice that uplink power increases faster than downlink for all three networks types. This is expected because sending data requires more power than receiving data for wireless data access [21]. LTE has the largest gap of $\alpha_{u} / \alpha_{d}=$ 8.44 among three network types. This is largely because $\alpha_{d}$ for LTE is quite small. For 3G, both $\alpha_{u}$ and $\alpha_{d}$ are larger than LTE. $\beta$ is the base power when throughput is 0 , with the ranking of LTE > $3 \mathrm{G}>\mathrm{WiFi}$. This is consistent with the tail base power comparison in Table 3 . We notice that $\beta$ is slightly higher than the tail base for all networks types. This is possibly because of the overhead of switching transmitters or receivers into high speed mode.

For simultaneous uplink and downlink transfers, given that transmitters and receivers are separate, we conjecture that the power level $(\mathrm{mW})$ is given by the following formula:

$$
P=\alpha_{u} t_{u}+\alpha_{d} t_{d}+\beta
$$

To validate this conjecture, we measure the power levels for concurrent uplink and downlink transfers in Figure 11 Assume total throughput $t=t_{u}+t_{d}$ and the ratio of uplink throughput $\epsilon=t_{u} / t$ :

$$
P=\alpha_{u} t_{u}+\alpha_{d} t_{d}+\beta=\left(\alpha_{u}-\alpha_{d}\right) t \epsilon+\alpha_{d} t+\beta
$$

When $t$ is a constant, $P$ grows linearly with $\epsilon$ and the slope is $\left(\alpha_{u}-\right.$ $\left.\alpha_{d}\right) t$. Figure 11 shows two curves of $t=1 \mathrm{Mbps}$ and $t=2 \mathrm{Mbps}$, both having a strong linear pattern and the slope is less than $5 \%$ off the expected value.

\subsection{Energy efficiency for bulk data transfer}

To compare the power efficiency of different networks in the wild, we use bulk data transfer experiments to measure energy per bit. Perrucci et al. [26] measure energy per bit for 3G and WiFi with a fixed bulk size. In addition to taking LTE into consideration, we vary the bulk size to cover more possible network usage scenarios. Figure 12 shows the measured energy per bit in $\mu \mathrm{J} /$ bit $\left(10^{-6}\right.$ Joule/bit $)$ with different bulk data size. All data is randomly generated so that there is no chance for caching. We do not include promotion or tail energy but instead focus on data transfer energy. Given that signal strength and peak data rate on wireless 

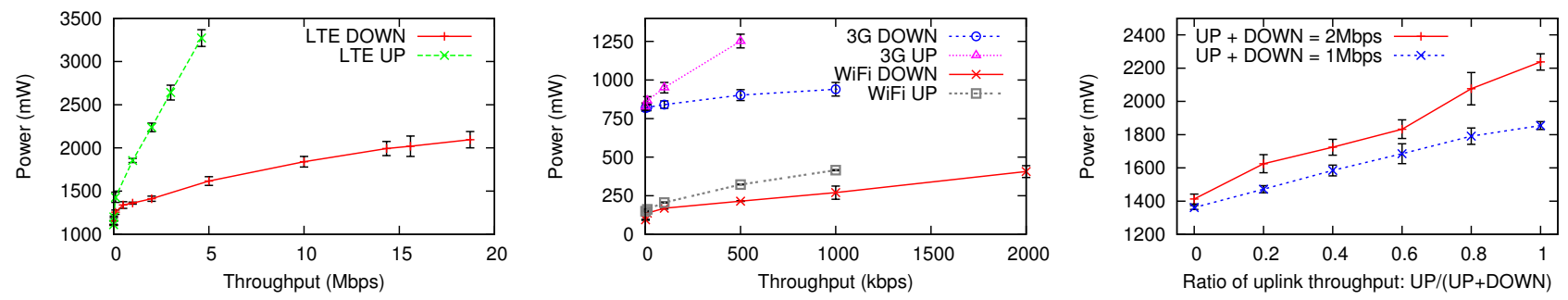

Figure 9: Power-throughput curve for LTE net- Figure 10: Power-throughput curve for 3G and Figure 11: Power of simultaneous uplink and work.

WiFi.

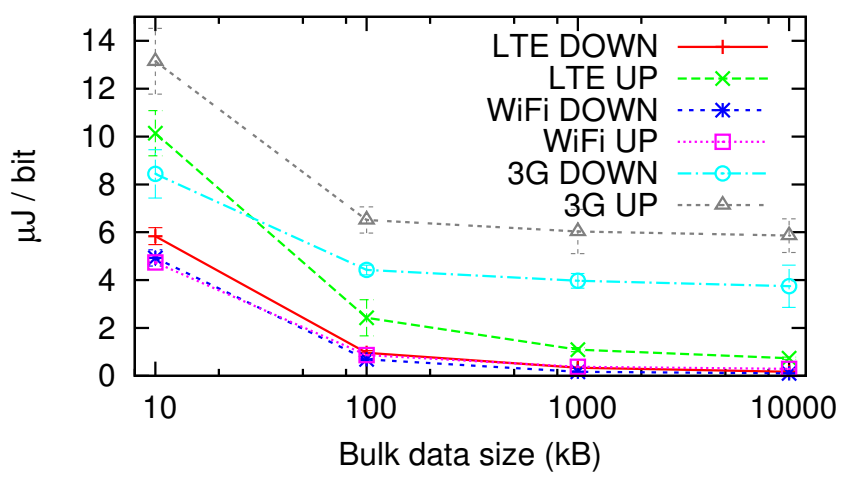

Figure 12: Energy per bit for bulk data transfers.

networks fluctuates, both affecting energy per bit, our measurement only serves as a sampled view for the energy efficiency of different networks.

First, energy per bit decreases as bulk data size increases, largely because with a small data size, throughput does not reach link capacity due to TCP slow start. We also observe that LTE's energy per bit in downlink is comparable with $\mathrm{WiFi}$, although the absolute power level of LTE is much higher than WiFi. This is due to high downlink throughput for LTE at our test location, even compared with the WiFi network. Similarly, for LTE uplink, it drops from $10 \mu \mathrm{J} /$ bit to less than $1 \mu \mathrm{J} /$ bit as bulk data size increases. With bulk data size of 10MB, LTE consumes 1.62 times the energy of WiFi for downlink and 2.53 for uplink. With lowest throughput, $3 \mathrm{G}$ has the worst energy efficiency for large data transfer, e.g., for downloading $10 \mathrm{MB}$ data, $3 \mathrm{G}$ requires 21.50 times the energy of LTE and 34.77 times the energy of WiFi, and for uplink, 7.97 times of LTE and 20.16 times of WiFi.

\subsection{Power model validation}

To validate the LTE power model and the trace-driven simulation (\$3.3.2), we compare measured energy (measured from LTE Phone) with simulated energy for case study applications. Table 5 contains the sample application usage scenarios described in $\$ 3.4$ The error rate is consistently less than $6 \%$, with the largest error rate from Website $Y$, which includes heavy JavaScript execution and HTML rendering. Since our power model focuses on radio power and ignores the impact of CPU, for Website $\mathrm{Y}$, the total energy usage is slightly underestimated.

The error rate is increased if the impact of downlink and uplink throughput is ignored, i.e., assuming $\alpha_{u}=\alpha_{d}=0$. However, the increase is not significant, at most $1.5 \%$. This is because for

\begin{tabular}{|c|c|c|c|}
\hline App & $\begin{array}{c}\text { Measured } \\
\text { energy }(\mathrm{J})^{1}\end{array}$ & $\begin{array}{l}\text { Simulated } \\
\text { energy }(\mathrm{J})^{1}\end{array}$ & Error \\
\hline Website $\mathrm{G}^{3}$ & 24.77 & 24.37 & $-1.61 \%\left(-2.06 \% \%^{2}\right)$ \\
\hline Website $\mathrm{Y}^{3}$ & 31.84 & 30.08 & $-5.53 \%(-7.04 \%)$ \\
\hline YouTube & 21.81 & 21.14 & $-3.07 \%(-4.17 \%)$ \\
\hline NPR News & 24.65 & 24.37 & $-1.12 \%(-1.70 \%)$ \\
\hline Market & 38.64 & 38.03 & $-1.58 \%(-3.03 \%)$ \\
\hline
\end{tabular}

Table 5: LTE power model validation.

these web-based applications, network throughput is low due to small object size $(\$ 7.2$ ). For other applications, such as video/audio streaming and file download, we expect to see a larger gap in error rate if the impact of downlink/uplink is ignored.

In this section, in addition to comparing energy per bit in bulk data transfer for different networks, we construct a new LTE power model and validate its accuracy, which is the basis for the following analysis.

\section{USER TRACE BASED TRADEOFF ANAL- YSIS}

In this section, we apply the LTE power model to UMICH data set and compare energy efficiency with $3 \mathrm{G}$ and $\mathrm{WiFi}$, with detailed break down of the total energy. We then study the tradeoff of configuring different LTE parameters via our analysis framework.

\subsection{Energy efficiency comparison}

We use the UMICH data set to simulate the LTE, WiFi and $3 \mathrm{G}$ model. Assume that the simulated energy usage for LTE, WiFi and $3 \mathrm{G}$ power model is $E_{l t e}, E_{\text {wifi }}$ and $E_{3 g}$, respectively, the energy ratio of LTE/WiFi is defined as $E_{l t e} / E_{\text {wifi }}$, and that for $3 \mathrm{G} / \mathrm{WiFi}$ is calculated as $E_{3 g} / E_{\text {wifi } i}$. With the same traces, we can make fair comparison among different power models to understand their energy efficiency.

In Figure 13 we compare the energy efficiency of different networks for the 20 users both individually and in aggregate (summing up for all users). We first observe that LTE power model consumes significantly more energy than WiFi. The ratio of LTE/WiFi ranges from 16.9 to 28.9 and the aggregate ratio for all users is 23.0. Notice that the gap between LTE and WiFi is larger compared with the bulk data transfer experiments in $\$ 5.3$ This is because, for bulk data transfer, LTE's high throughput could compensate the low energy efficiency, compared with real traces, which do not saturate the link capacity. Second, for $3 \mathrm{G} / \mathrm{WiFi}$ ratio, the range is between 10.8 


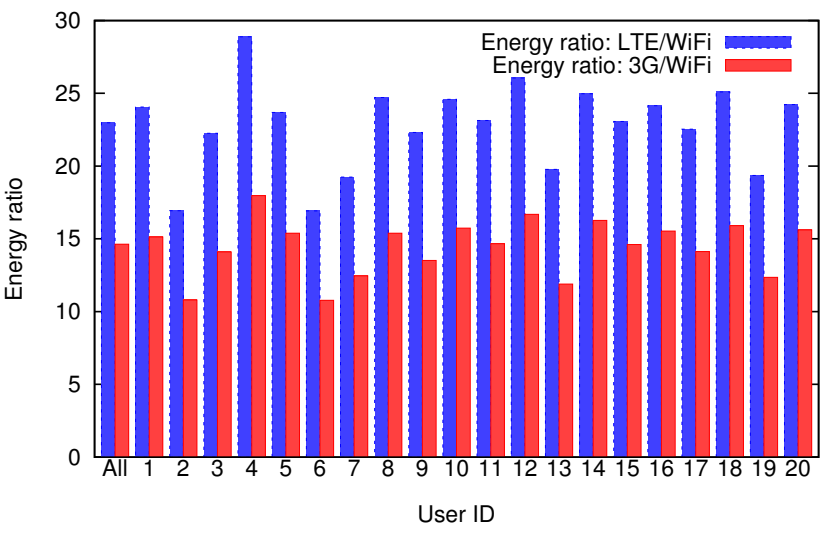

Figure 13: Power model simulation: energy ratio.

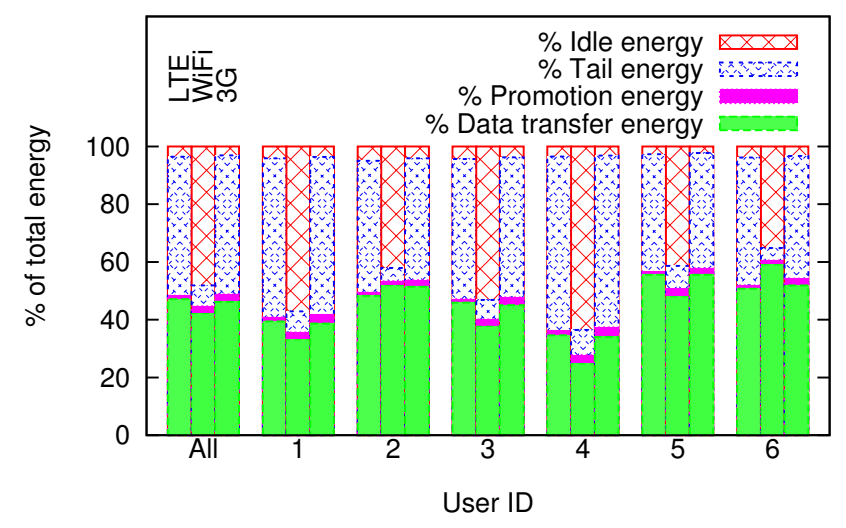

Figure 14: Break down of energy usage in analysis.

and 18.0, and in aggregate, $3 \mathrm{G} / \mathrm{WiFi}$ ratio is 14.6 , lower than LTE. In summary, the energy efficiency for LTE is lower than $3 \mathrm{G}$, with WiFi having a much higher energy efficiency. Notice that in this work, we do not consider AP association energy for WiFi networks due to the lack of such information, so $E_{w i f i}$ is an underestimate. However, we believe that AP association does not happen very often and the impact is not significant.

\subsection{Energy consumption break down}

To further understand the energy consumption, with the methodology discussed in $\$ 3.3 .2$ it is decomposed into promotion energy, data transfer energy, tail energy, and idle energy in Figure 14 with results for aggregate analysis and 6 sample users. These energy values are calculated including the UE base power.

Promotion energy contributes to a small portion $(<4 \%)$ of the total energy for all networks, i.e., in aggregate, its contribution is only $1.2 \%, 2.5 \%$ and $2.6 \%$ for $\mathrm{LTE}, \mathrm{WiFi}$, and $3 \mathrm{G}$, respectively. In terms of idle energy, WiFi has significantly higher percentage than LTE and 3G, despite small average power difference at idle state across networks, i.e., $31.1 \mathrm{~mW}^{3}$ for LTE, $13.0 \mathrm{~mW}$ for WiFi and $15.3 \mathrm{~mW}$ for $3 \mathrm{G}$. This is explained by WiFi's smaller total energy, making its idle energy contribution relatively higher.

Aggregate data transfer energy percentage is $47.1 \%, 42.1 \%$ and

\footnotetext{
${ }^{3}$ The idle power for LTE network is calculated as the average power of a DRX cycle in RRC_IDLE, and similarly for WiFi and 3G networks.
}

$46.2 \%$ for LTE, WiFi and 3G, respectively. The variation across users is high, e.g., for LTE network, it ranges from $22.0 \%$ to $62.3 \%$, due to traffic pattern differences across users.

Surprisingly, the biggest energy component for LTE network is tail, rather than data transfer. The average tail energy for LTE and $3 \mathrm{G}$ is $48.2 \%$ and $48.1 \%$ respectively compared to $7.2 \%$ for $\mathrm{WiFi}$. Our observation for $3 \mathrm{G}$ is consistent with previous study [18]. Combined with high data transfer energy due to the higher power levels, tail energy lowers the energy efficiency of LTE and 3G compared to $\mathrm{WiFi}$.

\subsection{Impact of LTE parameters}

Similar to $\$ 6.1$ we use WiFi traces in the UMICH data set to study the impact of LTE parameter configuration on radio energy $E$, channel scheduling delay $D$, and signaling overhead $S . E$ is the simulated total energy consumed by UE. Since most energy is consumed by radio interfaces, with display energy excluded, we approximate this total energy as radio energy. $D$ is the sum of scheduling delay for all packets, resulting from two factors: waiting for the next DRX cycle's on duration for a downlink packet and waiting for $T_{\text {pro }}$ during RRC_IDLE to RRC_CONNECTED promotion. $S$ is the overhead of the LTE network for serving this UE. $S$ has different definitions in our study given that different parameters studied affect signaling load in different ways, e.g., $T_{\text {tail }}$ affects mostly the state promotions, and $T_{p i}$ affects the total on duration in RRC_IDLE. $D$ and $E$ directly affect end users via application delay and battery life time; $S$ is of interest to cellular ISPs for supporting large user base at low cost.

\subsubsection{Impact of LTE tail timer $T_{\text {tail }}$}

In Figure 15, we study the impact of LTE tail timer $T_{\text {tail }}$, with $S$ defined to be the total number of RRC_IDLE to RRC_CONNECTED promotions. $T_{\text {tail }}$ varies from 1 second to 30 seconds. $T_{D}$ is the default configuration of 11.58 seconds for $T_{\text {tail }}$ in the measured LTE network with the corresponding values of $E, D$ and $S$ calculated as $E_{D}, D_{D}$ and $S_{D}$, respectively. When $T_{t a i l}$ is set to be $T^{\prime}$, similarly, we get $E^{\prime}, D^{\prime}$ and $S^{\prime}$. The change relative to the default setting is calculated as $\Delta(E)=\left(E^{\prime}-E_{D}\right) / E_{D}$, and similarly for $\Delta(D)$ and $\Delta(S) . \Delta$ s for each $T_{\text {tail }}$ values are plotted in Figure 15 and they are all 0 at $T_{D}$.

As expected, a larger $T_{\text {tail }}$ value reduces both $D$ and $S$, at the cost of $E$. We observe that $\Delta(S)$ and $\Delta(D)$ curves are almost identical, since most channel scheduling delay results from idle to active promotions. In addition, the impact of $T_{\text {tail }}$ on the radio energy $E$ is significant. For example, when $T_{\text {tail }}$ is set to be 30 seconds, total radio energy $E$ increases by $55 \%$, while for $T_{\text {tail }}=$ 1 second, $E$ decreases by $65 \%$, at the cost of $143 \% \Delta(D)$ and $169 \% \Delta(S)$. This indicates that $T_{\text {tail }}$ is a very important parameter for LTE network.

In previous study [28], traffic bursts attribute to low efficiencies of radio resource and energy utilization in 3G UMTS network, due to tail time. Given that LTE has similar tail time, we analyze the bursts in the UMICH data set. We follow the same definition of a burst as in [28], which is a sequence of consecutive packets whose inter-arrival time is less than a threshold $\delta$, with $\delta$ set to be 1.5 seconds, since it is longer than common cellular RTTs [24]. From ISP's perspective, $T_{\text {tail }}$ should not be smaller than majority of the inter-burst delays; otherwise, a larger number of promotions would be triggered.

We study the inter-burst delay in the $3 \mathrm{G}$ and $\mathrm{WiFi}$ traces of UMICH data set in Figure 19 For 3G traces, 67.3\% inter-burst delays are smaller than the default value of $T_{\text {tail }}$ (11.58 seconds), and $55.4 \%$ for WiFi traces. Compared with WiFi traces, $3 \mathrm{G}$ traces 

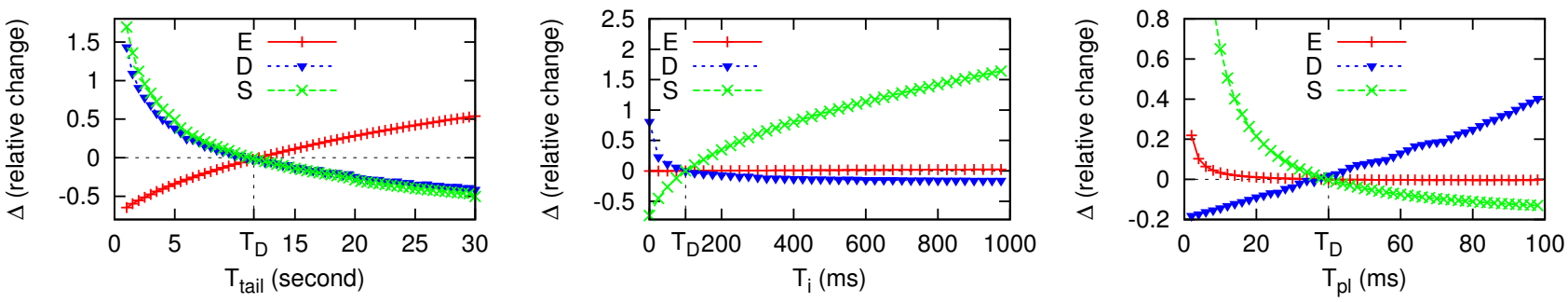

Figure 15: Impact of the LTE tail timer Figure 16: Impact of the DRX inactivity timer Figure 17: Impact of the DRX cycle in $T_{\text {tail }}$.

$T_{i}$.
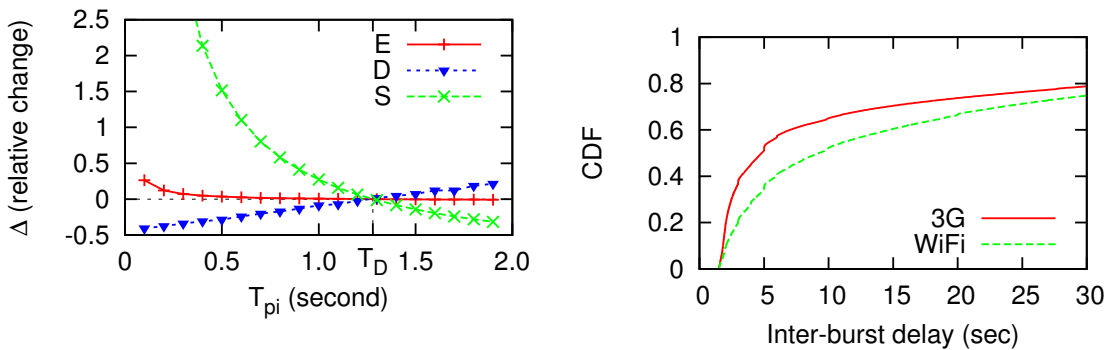

Figure 18: Impact of DRX cycle in Figure 19: CDF of Inter-burst delay for Figure 20: CDF of Inter-packet delay for RRC_IDLE $\left(T_{p i}\right)$.
UMICH data set.
RRC_CONNECTED $\left(T_{p l}\right)$.

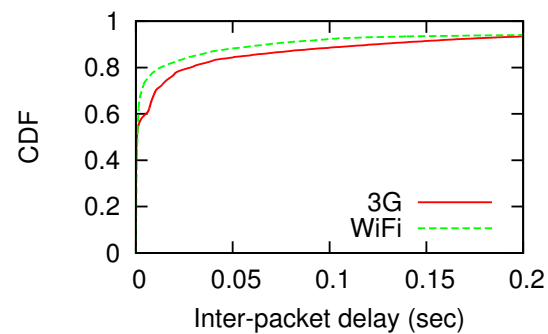

have smaller inter-burst delays. We also observe the inter-burst delay distribution for different users have non-negligible differences. This makes us believe that a per-UE based dynamic $T_{\text {tail }}$ configuration mechanism, adapting to traffic patterns, provides a better chance for optimizing radio resource efficiency and balancing energy utilization. Notice that this approach is inside the radio access network and transparent to UE. Previous study on 3G UMTS network also points out two possible approaches to deal with the tail effect with support from UE, i.e., applications proactively alter traffic patterns based on the state machine behavior without interaction with the network, or cooperate with the radio access network in allocating radio resources by fast dormancy [11.13]. These approaches remain to be applicable solutions for the tail problem in LTE.

\subsubsection{Impact of DRX inactivity timer $T_{i}$}

Figure 16 shows the tradeoff of setting DRX inactivity timer $T_{i}$. Signaling overhead $S$ is defined as the sum of the continuous reception time and DRX on durations in RRC_CONNECTED, since during these time slots, UE exchanges control messages with eNB (base station of LTE network) [30]. A larger $T_{i}$ keeps UE in continuous reception longer and reduces the scheduling delay for downlink packets, i.e., no DRX waiting time, at the cost of higher energy usage, since continuous reception has higher power level than DRX idle state. $T_{D}$ is the default value $100 \mathrm{~ms}$.

We observe that $T_{i}$ has negligible impact on $E$, with only $2 \%$ $\Delta(E)$ as $T_{i}$ is set to be 1 second. Different from $E, S$ is significantly affected by $T_{i}$. For $D$, when $T_{i}$ is set to be small than $100 \mathrm{~ms}, \Delta(D)$ grows up to $80 \%$. However, even when $T_{i}$ is set to be as large as 1 second, $D$ is only reduced by $16 \%$. This can be partly explained by Figure 20 that $88.6 \%$ packet pairs has $\leq 100 \mathrm{~ms}$ inter-packet delay for $3 \mathrm{G}$ traces and $92.3 \%$ for WiFi traces in the UMICH data set. Decreasing $T_{i}$ causes more packets arrive outside of continuous reception, which may experience DRX delay. Hence similar to $T_{\text {tail }}$, the setting of $T_{i}$ is also affected by the traffic pattern, i.e., inter-packet delay distribution, which may differ across users.

\subsubsection{Impact of DRX cycle in RRC_CONNECTED}

In RRC_CONNECTED, Short DRX cycle timer $T_{i s}$ determines the transition from Short DRX to Long DRX. Given that we already vary the DRX cycle to understand its impact, for simplicity, we assume $T_{i s}$ to be 0 , i.e., the DRX cycle in RRC_CONNECTED refers to Long DRX cycle $T_{p l}$.

In Figure 17 is defined similarly as in Figure 16 i.e., the sum of continuous reception time and DRX on durations. $T_{D}$ is $40 \mathrm{~ms}$, the default setting for $T_{p l}$. First, we notice that compared with $T_{D}$, when $T_{p l}$ is set to be $100 \mathrm{~ms}$, energy saving $\Delta(E)$ is minor $(0.2 \%)$ with $13.0 \%$ reduction in $S$, at the cost of $40 \%$ increase in $D$. So $T_{p l}$ is not recommended to be set much higher than $T_{D}$ unless ISP prioritizes $S$ reduction. When $T_{p l}$ is set to be a small value, for example $2 \mathrm{~ms}, E$ increases by $21.9 \%$ and $D$ reduces by $18.1 \%$, with a significant increase in $S$ of $412.9 \%$. Especially for ISPs with fast growing LTE user volume and congested radio access network, DRX cycle in RRC_CONNECTED is not recommend to be set to too small values. We omit the simulation results for Short DRX cycle $T_{p s}$ and Short DRX timer $T_{i s}$, since reducing $T_{p s}$ or increasing $T_{i s}$ has similar effect of decreasing $T_{p l}$, i.e., resulting in a shorter average DRX cycle, and their impact is similar as in Figure 17

\subsubsection{Impact of DRX cycle in RRC_IDLE}

Similarly to RRC_CONNECTED, we study the impact of DRX cycle in RRC_IDLE. Under this setting, signaling overhead $S$ is defined to be the total on duration of DRX cycles in RRC_IDLE, during which control messages are exchanged between UE and eNB [30]. In Figure 18 the default setting $1.28 \mathrm{~s}$ is shown as $T_{D}$. The impact of $T_{p i}$ on $E$ is not significant when $T_{p i}$ is larger than $T_{D}$, with $0.7 \%$ energy saving when $T_{p i}$ set to be $2.0 \mathrm{~s}$, which also brings in $21.3 \%$ increase in $D$ and $31.7 \%$ reduction in $S$. When $T_{p i}$ is set to 


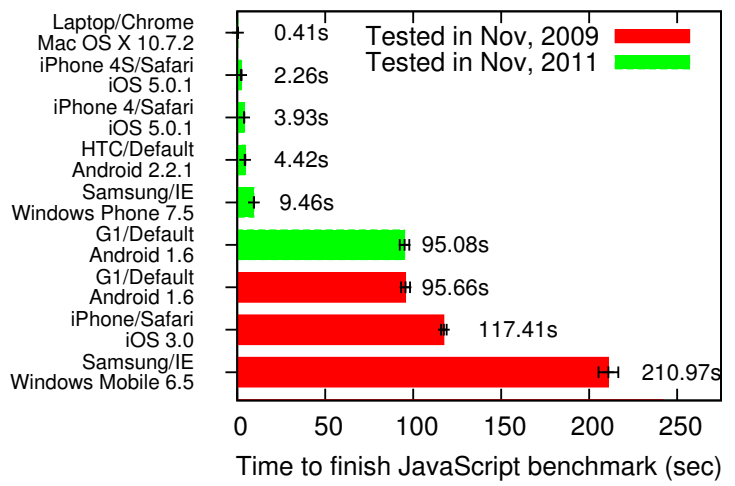

Figure 22: JavaScript execution speed comparison.

be $100 \mathrm{~ms}$, the energy overhead is $26.2 \%$, with $40.7 \%$ reduction in $D$ and up to $1147.2 \%$ increase in $S$.

Similar to $T_{p l}, T_{p i}$ causes significant signaling overhead when set to be too small. Default values for both $T_{p l}$ and $T_{p i}$ are reasonable settings and adjustment around the default configurations results in moderate impact on channel scheduling delay and negligible impact on radio energy.

In this section, we observe that LTE consumes up to 23 times the total energy of WiFi in the simulation, and the tail problem in 3G UMTS network is still a key contributor for the low energy efficiency for LTE. Similar to $3 \mathrm{G}$ network, $T_{\text {tail }}$ remains a key parameter for LTE. In addition to the UE-participated approaches including proactive traffic shaping and fast dormancy proposed by previous study [27], we discuss a possible approach to solve the tail problem in LTE network, which dynamically adapts $T_{\text {tail }}$ to traffic pattern at a per-UE base, being transparent to UE. Similar observations are shared by the other LTE parameters and we leave the feasibility study of such dynamic parameter configuration framework for LTE as future work.

\section{APPLICATION PERFORMANCE IMPACT}

In this section, we try to identify the new bottleneck of webbased smartphone applications in LTE and start with comparing the processing power of contemporary smartphones.

\subsection{JavaScript execution}

JavaScript execution is an important component in web transactions and we use it as a sampled view of UE's processing power. In 2009, we found that the JavaScript execution speed for smartphone browsers could be up to 20 80 times slower than desktop browsers [24]. We revisit the JavaScript execution experiments in this study, as shown in Figure 22 We consistently use the same benchmark [8] (version 0.9) to quantify the JavaScript execution speed. In Figure 22, the test results on the same G1 phone in 2009 and in 2011 are very close, validating that the benchmark of that version has not changed since 2009.

From 2009 to 2011, iOS has a speedup of 29.88 for iPhone 4 and 51.95 for iPhone 4S, while 21.64 for Android and 22.30 for Windows Phone. And the gap between smartphone and computer has dropped to 5.5 23.1 times in JavaScript execution speed. Possible reasons for this improvement include fast CPU, larger memory, and better OS and application software for smartphones.

\subsection{Application case study}

Along with improvements in mobile client processing power,

\begin{tabular}{|c|c|c|c|c|}
\hline App & Total payload & Max obj size & \# objects & Avg size \\
\hline Website G & $160.7 \mathrm{~KB}$ & $87.6 \mathrm{~KB}$ & 11 & $14.6 \mathrm{~KB}$ \\
\hline Website Y & $509.9 \mathrm{~KB}$ & $124.2 \mathrm{~KB}$ & 59 & $8.6 \mathrm{~KB}$ \\
\hline YouTube & $449.1 \mathrm{~KB}$ & $109.4 \mathrm{~KB}$ & 33 & $13.6 \mathrm{~KB}$ \\
\hline NPR News & $306.2 \mathrm{~KB}$ & $169.5 \mathrm{~KB}$ & 9 & $34.0 \mathrm{~KB}$ \\
\hline Market & $599.7 \mathrm{~KB}$ & $145.4 \mathrm{~KB}$ & 17 & $35.3 \mathrm{~KB}$ \\
\hline
\end{tabular}

Table 6: HTTP object statistics.

LTE technology significantly increased network speed. So we perform a case study of a few popular applications to understand the impact of network speed and processing power enhancement on user-perceived performance.

With the measurement methodology discussed in $\$ 3.4$, we compare loading time, CPU usage, and energy consumption for five application usage scenarios in Figure 21. These usage scenarios are representative for web browsing and other similar web-based applications, different from audio and video streaming applications.

First, loading time comparison shows that LTE and WiFi have comparable user-perceived delay for the studied scenarios, and $3 \mathrm{G}$ lags behind with 50\% 200\% larger response time. The application loading time for LTE slightly lags behind WiFi. On one hand, given that promotion delay is counted into the loading time, LTE has larger $T_{\text {pro }}$. On the other hand, though LTE has faster throughput than WiFi with throughput tests, its RTT is slightly larger. To understand why RTT matters more than throughput for the usage scenarios in Figure 21 we analyze the object size statistics for different applications in Table 6

In Table 6 across all scenarios, the maximum object size is $169.5 \mathrm{~KB}$ for NPR News and the average object size is at most 35.3KB for Android market. Especially for Website $\mathrm{Y}$, there are 59 HTTP objects with an average size of only 8.6KB. Due to TCP slow start, these objects are far from saturating the link capacity for either LTE or WiFi. Persistent TCP connections are observed to transfer multiple web objects; however, no HTTP pipelining is observed. We also observe up to 5 parallel TCP connections to different remote servers and even to the same remote server; however the average low throughput, e.g., 364.2kbps for Website Y, indicates that the RTT is more critical than available bandwidth for these web-based applications in LTE and WiFi.

We observe that average CPU usage for $3 \mathrm{G}$ network is relatively low ranging from $35.5 \%$ to $70.8 \%$, with an average of $57.7 \%$. CPU usage for LTE is between $68.8 \%$ and $84.3 \%$, averaging at $79.3 \%$, compared with WiFi's $78.2 \% \sim 93.0 \%$ and $87.1 \%$ as average. This comparison implies that the gap between WiFi and cellular network has narrowed because of LTE's better network performance. For $3 \mathrm{G}$ network, network performance appears to be the bottleneck, resulting in underutilized processing power. Given that the average CPU usage for LTE and WiFi is already $80 \% \sim 90 \%$, further performance optimization relies more on processing speed improvement and OS/application software enhancement, compared with network improvement. Work such as CloneCloud [20] on offloading computation into the cloud is one such promising direction.

In terms of energy usage, WiFi clearly has significantly higher energy efficiency for the usage scenarios studied, similar to previous discussions. 3G consumes 5.5 7.8 times the energy of WiFi, with $20.9 \% \sim 55.6 \%$ of the total energy due to tail. While for LTE, energy usage is $5.4 \sim 12.0$ times of $\mathrm{WiFi}$, with $32.2 \% \sim 62.2 \%$ tail energy. We notice that, most of these applications keep some of the existing TCP connections open even after the initial loading; so that if user generates subsequent requests, these connections could be reused. Similar to previous work [28], we observe that appli- 


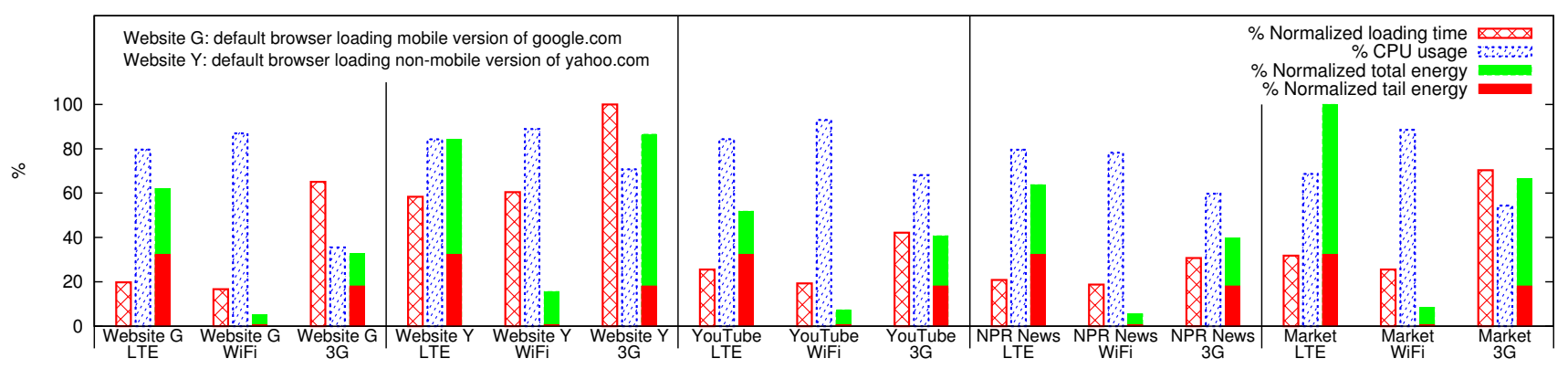

Figure 21: Loading time, CPU usage and energy consumption analysis on mobile applications.

* The loading time is normalized by the maximum loading time observed in this figure, and similarly for total energy.

cations remain idle after the initial loading and a few TCP control packets are generated by UE to close the existing applications a few seconds later, such as the packets seen at time 10.5 seconds in Figure 4 which reset $T_{\text {tail }}$ and further increase the high-power tail length.

In this section, we observe that contemporary smartphones have reduced gap with desktop computers in terms of processing power. However, for web-based applications, downloading mostly small sized objects, the performance bottleneck is still at the UE processing side, given the significant improvement in network speed for LTE.

\section{RELATED WORK}

We summarize related work in three categories below.

Measuring 2G and 3G networks. Prior study [18] investigates the impact and the optimization of tail effects in $2 \mathrm{G} / 3 \mathrm{G}$ networks. Previous work [27] characterizes the impact of RRC state machine on radio resources and energy by analyzing a dataset collected from a commercial UMTS network. Recent work [22] also investigates impact of traffic patterns on radio power management policy. Other measurement studies (e.g., 3GTest [24], LiveLab [31], and [23]) collect traces from real smartphone users, focusing on characterization at only IP and higher layers. None of them investigate resource management policy in 4G LTE networks.

Smartphone power modeling has also been investigated by previous empirical measurements. The Bartendr project [29] studies the relationship between signal strength and smartphone radio power usage. PowerTutor [35] collects power traces for individual hardware components under controlled experiments then uses multi-variable regression to compute the coefficients for a linear power model considering all hardware components. ARO [28] employs an approach similar to [35] but focusing only on radio power usage. It performs more fine-grained simulation of transmission queues to capture state transitions. Our LTE power model is also empirically derived, but it differs from all aforementioned models in two aspects. First, it considers DRX in RRC_CONNECTED, a new power management mechanism in LTE networks. Second, it further takes into account both uplink and downlink data rates, resulting in a more accurate estimation of the radio power consumption when the throughput is high, as is a common case in LTE networks.

DRX in 4G LTE networks. Zhou et al. [36 model the DRX mechanism as a semi-Markov process, with which they analytically study the effects of DRX parameters on the performance, as well as the tradeoff between the power saving and wake-up delay. Kolding et al. [25] also investigate the balance between throughput and power saving by changing the configuration of DRX parame- ters, using a web-browsing traffic model. Bontu et al. [19] further considers the impact of DRX parameters on different applications with various delay sensitivities. Wigard et al. compares a longDRX-only scenario and a scenario with both long and short DRX, in terms of throughput and power consumption. All above studies employing analytical models suffer from an inherent limitation: the expressiveness of an analytical model is quite limited and is unlikely to capture the characteristics of real-world traffic patterns using a statistical distribution with a few parameters. The existence of concurrent applications accessing the network further increases the difficulty of modeling the packet dynamics. In contrast, our work is the first empirical study to investigate the impact of the configurations of DRX parameters and tail timer. We overcome the above limitation by employing network traces from real smartphone users, thus more realistically revealing the complex tradeoffs incurred by various DRX parameters.

\section{CONCLUSION}

In this paper, we take one of the first steps in understanding performance and power characteristics of 4G LTE networks. With publicly deployed 4GTest, we observe that LTE has significantly higher downlink and uplink throughput, compared with $3 \mathrm{G}$ and even WiFi. To characterize power usage for LTE, we derive the first comprehensive power model of a commercial LTE network, with less than $6 \%$ error rate. By applying the power model to a comprehensive data set, UMICH, we identify that LTE is much less power efficient than $\mathrm{WiFi}$, and the key contributor is the tail energy, controlled by $T_{\text {tail }}$. In addition, via a case study, we identify the UE processing to be the new bottleneck for web-based applications in LTE networks.

Our work opens new research opportunities for the new LTE network, such as optimizing smartphone applications with tools similar to ARO [28] with the energy model derived in this paper, and optimizing LTE parameter configuration with UE-based approaches (e.g., fast dormancy) or network-based approaches to improve radio resource and energy efficiency.

\section{ACKNOWLEDGEMENTS}

This work is partly funded by NSF grants CNS-1059372, CNS1050157, CNS-1039657 and Navy grant N00014-09-1-0705. We would like to thank Zhiyun Qian, Doug Sillars and the shepherd Sharad Agarwal for their valuable comments on the paper. We would also like to thank the participants in our user study and anonymous reviewers whose comments helped improve the final version. 


\section{REFERENCES}

[1] 3GPP LTE. http://www. 3gpp.org/LTE.

[2] 4GTest.http://mobiperf.com/4g.html

[3] BU-353 USB GPS Receiver.http: //www.usglobalsat.com/p-62-bu-353-w.aspx

[4] MaxMind.http://www.maxmind.com

[5] Measurement Lab. http://www.measurementlab.net/

[6] MobiPerf (3GTest).http://mobiperf.com

[7] Monsoon power monitor.http://www.msoon.com/ LabEquipment/PowerMonitor

[8] SunSpider JavaScript Benchmark 0.9. http: / / www . webkit.org/perf/sunspider/sunspider.html

[9] TCPDUMP and LIBPCAP. http://www.tcpdump.org/

[10] 3GPP TR 25.813: Radio interface protocol aspects (V7.1.0), 2006.

[11] UE "Fast Dormancy" behavior. 3GPP discussion and decision notes R2-075251, 2007.

[12] 3GPP TR 25.913: Requirements for Evolved UTRA and Evolved UTRAN (V9.0.0), 2009.

[13] Configuration of fast dormancy in release 8. 3GPP discussion and decision notes RP-090960, 2009.

[14] 3GPP TS 25.304: User Equipment (UE) procedures in idle mode and procedures for cell reselection in connected mode (V10.2.0), 2011.

[15] 3GPP TS 36.211: Physical Channels and Modulation (V10.3.0), 2011.

[16] 3GPP TS 36.321: Medium Access Control (MAC) protocol specification (V10.3.0), 2011.

[17] 3GPP TS 36.331: Radio Resource Control (RRC) (V10.3.0), 2011.

[18] N. Balasubramanian, A. Balasubramanian, and A. Venkataramani. Energy Consumption in Mobile Phones: A Measurement Study and Implications for Network Applications. In IMC, 2009.

[19] C. S. Bontu and E. Illidge. DRX Mechanism for Power Saving in LTE. In IEEE Communications Magazine, 2009

[20] B.-G. Chun, S. Ihm, P. Maniatis, M. Naik, and A. Patt. CloneCloud : Elastic Execution between Mobile Device and Cloud. In Proceedings of 6th European Conference on Computer Systems (EuroSys 2011), 2011.

[21] S. Cui, A. J. Goldsmith, and A. Bahai. Energy-Efficiency of MIMO and Cooperative MIMO Techniques in Sensor Networks. In IEEE J. Sel. Areas Commun., vol. 22, no. 6, pp. 1089-1098, 2004.
[22] H. Falaki, D. Lymberopoulos, R. Mahajan, S. Kandula, and D. Estrin. A First Look at Traffic on Smartphones. In Proc. ACM SIGCOMM IMC, 2010.

[23] H. Falaki, R. Mahajan, S. Kandula, D. Lymberopoulos, and R. G. D. Estrin. Diversity in Smartphone Usage. 2010.

[24] J. Huang, Q. Xu, B. Tiwana, Z. M. Mao, M. Zhang, and P. Bahl. Anatomizing Application Performance Differences on Smartphones. In MobiSys, 2010.

[25] T. Kolding, J. Wigard, and L. Dalsgaard. Balancing Power Saving and Single User Experience with Discontinuous Reception in LTE. In ISWCS, 2008.

[26] G. Perrucci, F. Fitzek, and J. Widmer. Survey on Energy Consumption Entities on Smartphone Platform. In Vehicular Technology Conference, 2009.

[27] F. Qian, Z. Wang, A. Gerber, Z. M. Mao, S. Sen, and O. Spatscheck. Characterizing Radio Resource Allocation for 3G Networks. In IMC, 2010.

[28] F. Qian, Z. Wang, A. Gerber, Z. M. Mao, S. Sen, and O. Spatscheck. Profiling Resource Usage for Mobile Applications: a Cross-layer Approach. In MobiSys, 2011.

[29] A. Schulman, V. Navda, R. Ramjee, N. Spring, P. Deshpande, C. Grunewald, K. Jain, and V. N. Padmanabhan. Bartendr: A Practical Approach to Energy-aware Cellular Data Scheduling. In MobiCom, 2010.

[30] S. Sesia, I. Toufik, and M. Baker. LTE: The UMTS Long Term Evolution From Theory to Practice. John Wiley and Sons, Inc., 2009.

[31] C. Shepard, A. Rahmati, C. Tossell, L. Zhong, and P. Kortum. LiveLab: Measuring Wireless Networks and Smartphone Users in the Field. In HotMetrics, 2010.

[32] S. Sundaresan, W. de Donato, R. Teixeira, S. Crawford, and A. PescapÃí. Broadband Internet Performance: A View From the Gateway. In SIGCOMM, 2011.

[33] Z. Wang, F. X. Lin, L. Zhong, and M. Chishti. Why are Web Browsers Slow on Smartphones? In HotMobile, 2011.

[34] J. Wigard, T. Kolding, L. Dalsgaard, and C. Coletti. On the User Performance of LTE UE Power Savings Schemes with Discontinuous Reception in LTE. In ICC Workshops, 2009.

[35] L. Zhang, B. Tiwana, Z. Qian, Z. Wang, R. P. Dick, Z. M. Mao, and L. Yang. Accurate Online Power Estimation and Automatic Battery Behavior Based Power Model Generation for Smartphones. In CODES+ISSS, 2010.

[36] L. Zhou, H. Xu, H. Tian, Y. Gao, L. Du, and L. Chen. Performance Analysis of Power Saving Mechanism with Adjustable DRX Cycles in 3GPP LTE. In Vehicular Technology Conference, 2008. 